\title{
Perceptions of Video-Based Appointments from the Patient's Home: A Patient Survey
}

\author{
Matthew R. Gardner, MBA, MDes, ${ }^{1}$ Sarah M. Jenkins, MS, ${ }^{2}$ \\ Daniel A. O'Neil, MBA, MSIE, ${ }^{1}$ Douglas L. Wood, MD, ${ }^{1}$ \\ Barbara R. Spurrier, $M H A^{1}{ }^{1}$ and Sandhya Pruthi, $M D^{3}$ \\ ${ }^{1}$ Center for Innovation, ${ }^{2}$ Department of Health Sciences Research, \\ and ${ }^{3}$ Division of General Internal Medicine, Mayo Clinic, \\ Rochester, Minnesota.
}

\begin{abstract}
Background: We examined patient interest in a telehealth model in which the patient supplies the hardware and Internet connectivity to meet with a healthcare provider from his or her home via video call (video appointment). We hoped to understand prospectively the desirability, feasibility, and viability from the patient perspective. Materials and Methods: A phone survey was conducted of a random sample of patients who had been seen in the outpatient setting at a single institution. The sample was stratified by proximity to the local institution with oversampling for patients living outside a 120-mile radius. Results: Out of 500 total patients, 301 patients responded, and 263 met the inclusion criteria. Of those 263 respondents, 38\% indicated "very likely" to accept an invitation to see their provider via video, 28.1\% "somewhat likely," and 33.8\% "not at all likely." of respondents, 75\% have broadband, although only 36\% reported having a Web camera. The primary factors affecting willingness to participate in a video appointment include comfort in setting up a video call, age, and distance participants would have traveled for an in-clinic appointment. Conclusions: Patient survey data indicate that most patients are likely to be accepting of telehealth care to the home using video call and that most have the required technology. Nevertheless, there are still significant hurdles to effectively implement this adaptation of telehealth care as part of mainstream practice.
\end{abstract}

Key words: appointment, feasibility, patient survey, prospective, telemedicine, video, videoconference, video appointment, video call

\section{Introduction}

W

e have observed within practice a slowly increasing interest from both patients and providers to connect with each other over video call when they would have been otherwise meeting face to face. Telemedicine, like any service that we wish to grow and be sustainable, requires adequate supply and demand. From the supply perspective, one study by Grigsby et al. ${ }^{1}$ illustrated the many ways physician participation may limit the adoption of telemedicine, but the patient's interest and participation must also be a factor to consider. This study aimed to round out the factors that might affect patient adoption of telemedicine.

Many studies have measured the effectiveness of and satisfaction with specific telemedicine offerings in defined medical contexts. ${ }^{2-4}$ Other studies have focused on an age group. ${ }^{5,6}$ A review of similar survey studies pointed out the current challenges with patient satisfaction studies. ${ }^{7}$ Similar to our study, at least one study has requested input on a prospective service, but did so within a limited medical population, asking patients to rate use-case scenarios. ${ }^{8}$ In contrast, our study was designed to understand patient perceptions of an in-home video appointment as a particular service offering on a prospective basis with the hope to better understand general potential uptake across healthcare service lines. Because we aimed to get broad general representation across the institution, it was not limited by age, geography, or medical condition.

\section{Materials and Methods}

To get a broad representation of patients, we included patients seen by both primary care and specialist providers from a single academic institution in Minnesota that serves primary care, community, and regional specialty needs. For inclusion, the patients' last visit was required to be outpatient, under the assumption that an inpatient care episode aligned with acuity that would not be suitable for a video appointment. Because the institution serves the local and regional area, as well as subspecialty needs from a geographically much larger national and international draw, we randomly pulled 250 records of patients with addresses in the community and 11 surrounding counties (the "local" population) and 250 records for domestic patients with addresses outside those counties ("nonlocal"). All participants and all potential participants were over 18 years of age and had been seen at the institution between July 1, 2011, and July 31, 2012.

The survey questionnaire included questions about estimated future visits to the local institution, familiarity with video calling, patient technology ownership, preference for video appointments and factors informing those preferences, patient qualitative valuation of a video appointment, and fact collecting about the costs of travel to be evaluated in-person at the local institution (see Supplementary Fig. S1; Supplementary Data are available online at www.liebertpub.com/tmj). We augmented survey questions with demographic information accessed from the electronic medical record, including age, sex, and geographic location. The Institutional Review Board approved the study. Informed consent was obtained from all potential participants. 


\section{GARDNER ET AL.}

The telephone-based survey was conducted by survey administrators in a survey research call center. Eligible potential participants were contacted via telephone up to five times at varying times of the day and week. Nonrespondents included both those who opted out of the survey and those who never answered the phone.

\section{STATISTICAL ANALYSIS}

Responses for categorical survey items were summarized with frequencies and percentages, and continuous items were summarized with means and standard deviations (SDs) (or medians and ranges, where appropriate). Unadjusted comparisons were performed between groups (e.g., local versus non-local; likely versus unlikely to accept video visit) using chi-squared tests (or Fisher's exact tests) for categorical variables and using $t$ tests/analyses of variance (or Wilcoxon's rank-sum/Kruskal-Wallis test) for continuous variables. All analyses were performed using SAS version 9 (SAS Institute Inc., Cary, NC) or R. ${ }^{9}$ Values of $p<0.05$ were considered statistically significant.

\section{Results}

In total, 500 patients were included in the sample, and telephone attempts were made for 496 (4 deceased patients excluded) between September 5, 2012, and September 29, 2012. Of those contacted, 301 patients completed the survey (respondents). Of those not completing the survey, 114 were unable to be reached, 32 were unable to respond because of a barrier (e.g., language/ hearing barrier or mentally/physically unable), and 49 refused. Respondents who were not expecting to have further appointments with a provider from the institution were excluded from the analysis $(n=38)$, leaving 263 participants who met the criteria for analysis.

The mean age of all respondents $(n=301)$ was $57.9(\mathrm{SD}=18.1)$ years versus $53.0(\mathrm{SD}=20.4)$ years for nonrespondents $(p=0.006)$, indicating that respondents were older than nonrespondents. Respondents also tended to be more often female: $58.5 \%$ versus $48.2 \%$ of nonresponders ( $p=0.03$ ). Among the 263 patients ultimately included in the analysis, the average age was $57.9(\mathrm{SD}=17.7$; range, 18-93) years, and 151 (54.4\%) were female.

\section{TECHNOLOGY AND BEHAVIORAL PREVALENCE}

Most participants (84\%) in our sample had an endpoint device-a computer or smart device. Eighty-four percent owned a computer with Microsoft OS, and 12\% owned a Macintosh; only 4\% owned both. Most respondents (75\%) had access to broadband. Those who had broadband tended to be younger on average compared with those who did not (mean age, 55 versus 66 years, respectively; $p<0.0001$ ). Most (57\%) agreed that their current technology set-up would allow for a video call. About a third (38\%) said that they were comfortable setting up a video call on their own. Of the respondents, $53(20 \%)$ had participated in a video call before for either personal or business use. Of those, about half $(n=27,10 \%)$ used their home or personal technology.

\section{WILLINGNESS TO ACCEPT THE INVITATION AND KEY ASSOCIATIONS}

Thirty-eight percent of respondents said they were "very likely" to accept a video invitation, 28\% were "somewhat likely," and 38\% were "not at all likely." Those who had experienced video calling before were more favorable regarding a video's effectiveness to enable the same quality of care as a traditional visit. Of the participants who had participated in a video call before, 62\% agreed (or strongly agreed) that they could get the same quality of care as an in-person visit, whereas only $34 \%$ of the participants who had not participated before agreed with the statement ( $p=0.0004)$. Of those responding who had not participated in a video call, $86 \%$ wanted to speak with the provider in the traditional face-to-face encounter despite the cost of travel, whereas only 64\% of those who had experienced a call wanted to do so ( $p=0.0003)$. To a lesser degree, having participated in a call before also affected whether someone believed he or she would be able to communicate what he or she needed. Of those who had not participated in a video call before, 61\% agreed or strongly agreed that they would be able to communicate what they needed compared with $77 \%$ of those who had ( $p=0.03)$.

We posed 10 questions to capture prevalence of beliefs about video communications that had been exposed in previous qualitative discussions with patients. Of these questions, only one did not show a significant correlation with "likelihood to accept," and that was "I have a trusting relationship with my provider: (strongly) agree/ (strongly) disagree." The other questions in this set all were highly correlated with how participants responded (all $p<0.0001$ ). To determine the factors that were most likely to drive acceptance, the likelihood to accept a video appointment was compared with these items (4-point Likert scale) regarding thoughts/attitudes toward potential challenges (e.g., "I am confident that communications using video calling are private and secure") with this type of service. We estimated the Spearman rank correlation between each item versus the outcome (likelihood to accept). Each correlation was then plotted against the average response for each specific item (Fig. 1). This method allowed us to identify which specific items tended to be most associated with likelihood to accept while having lower than average attitudes toward specific, potentially modifiable, challenges. The probability of being "very likely" to accept a video appointment was compared between selected patient characteristics using multivariable logistic regression.

Being an employee at the local clinic made no significant difference regarding how participants responded to the likelihood of accepting a video appointment, and neither did "ruralness" of where participants lived, as defined by a census-based analysis (rural-urban commuting area codes) of proximity to metropolitan areas and traffic flow into those areas. ${ }^{10}$ Neither gender nor type of computer owned made a significant difference in willingness to participate in a video appointment.

\section{KEY DRIVERS AND PREDICTIVE MODELING}

After analysis of several potentially associated factors, we determined that there were three factors that were most closely associated 


\section{PATIENT PERCEPTIONS OF IN-HOME VIDEO APPOINTMENTS}

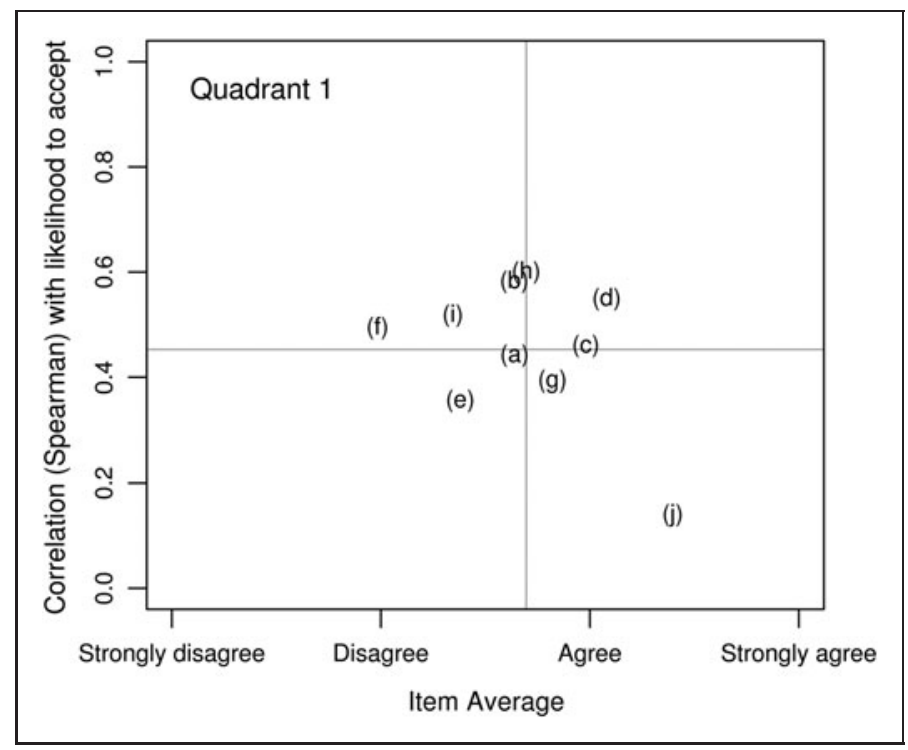

Fig. 1. Likelihood to accept a video appointment versus patientvoiced considerations in response to the following questions: (a) = My current technology set-up would allow for a video call; (b) = I am confident that I would be able to connect based on my level of computer experience; (c) =I am confident that communications using video calling are private and secure; $(d)=$ Although family/roommates could be exposed to information about my health, I am comfortable having an encounter with a medical provider in my home; (e)= Travel costs to visit [institution] are greater than the costs to purchase any tech or Internet service I don't yet have in place for video calling; $(f)=$ Considering my cost of travel, I still prefer to speak with my provider face to face [REVERSE CODED]; $(g)=$ In my case, I believe the provider is able to do his/her job even if they aren't able to conduct a physical exam every appointment; $(h)=I$ can communicate everything I need to in a video appointment; (i) = I can get the same quality of care from a video appointment as from an in-person visit; and $(j)=I$ have a trusting relationship $\mathrm{w} / \mathrm{my}$ [institution] providers. Note that the horizontal gray line indicates the average correlation, and the vertical gray line indicates the average "agreement." Therefore, quadrant 1 identifies challenges that have higher than average correlation with "likelihood to accept," while having lower than average "agreement."

with likelihood to accept an invitation to participate in a video appointment: comfort level in setting up for a video call, age, and distance from the medical facility.

Comfort level in setting up a video call. Those who were very likely to accept were most comfortable (66\%) compared with those who were somewhat likely to accept (37\%) or not at all likely to accept $(9 \%)(p<0.0001)$. Several other questions on the survey were also associated with comfort level; in general, comfort level was highest among those with more access to and experience with technology and those with more confidence in the video-calling process and understanding security issues.

Age. Those with a willingness to accept a video appointment had a mean age of 55.4 years, and the mean age was 64.1 years for those not at all likely to accept ( $p=0.0002$ ). Age also had a larger effect among those who were comfortable: increasing age was actually associated with a higher likelihood to accept an invite. Among those who were not comfortable, the likelihood to accept the invite decreased as age increased, although this was not significant.

Distance traveled. Those who had longer travel times were more likely to accept a video invite. This effect was most pronounced among those who were comfortable in setting up a video call.

A final model was determined to include comfort level, age, and total travel minutes, as well as an interaction between comfort levels and travel minutes. The predicted probabilities from this model were summarized graphically. Figure 2 represents the regression model predicting the probability of being "very likely" to accept an invite according to those factors.

\section{VALUE-BASED CONSIDERATIONS}

Few participants (14.4\%) indicated that a video appointment was of more value than a face-to-face appointment when an exam was not needed, although nearly half of respondents (46.7\%) indicated that it was of equal value. As would be expected, those who were more likely to accept the video appointment were also more likely to think a video appointment was of equal or greater value than a faceto-face appointment $(p<0.0001)$.

If insurance would not cover video appointments, 75.5\% of respondents indicated that it was not at all likely that they would accept a video appointment, whereas only $5.1 \%$ answered "very likely." This differed by whether or not the participant was local ( $p=0.0002)$; non-local participants were more accepting of a video appointment despite insurance not covering it, with 8.4\% "very likely" and 27.7\% "somewhat likely" (versus 2.2\% and 12.3\% for local participants, respectively). Almost uniformly, participants who reported having to take an economic loss, or make work, pet, or child care arrangements, were more likely to also have indicated that they were very likely to accept a video appointment.

Nineteen percent of our sample (29\% local, 7\% non-local) indicated that their last visit to the local institution involved one provider and required no additional tests. This reflects the patient population for whom a video visit would potentially be feasible and most beneficial. Among the 48 participants (39 were nonlocal, 9 were local) who saw just one provider and had no additional services in their last visit, 20 (41.7\%) were somewhat likely to accept a video invite, and 15 (31.3\%) were very likely. Therefore $74.4 \%$ of this subset (48 participants) are somewhat or very likely to accept.

\section{Discussion}

In our patient survey study we observed that most participants were likely to accept telehealth care at home by utilizing video appointment and that most had the required technology. For many users, a video appointment with their provider might be their first video call, and even if they have participated in a call, it may have been in a supported environment such as the workplace. Because 


\section{GARDNER ET AL.}

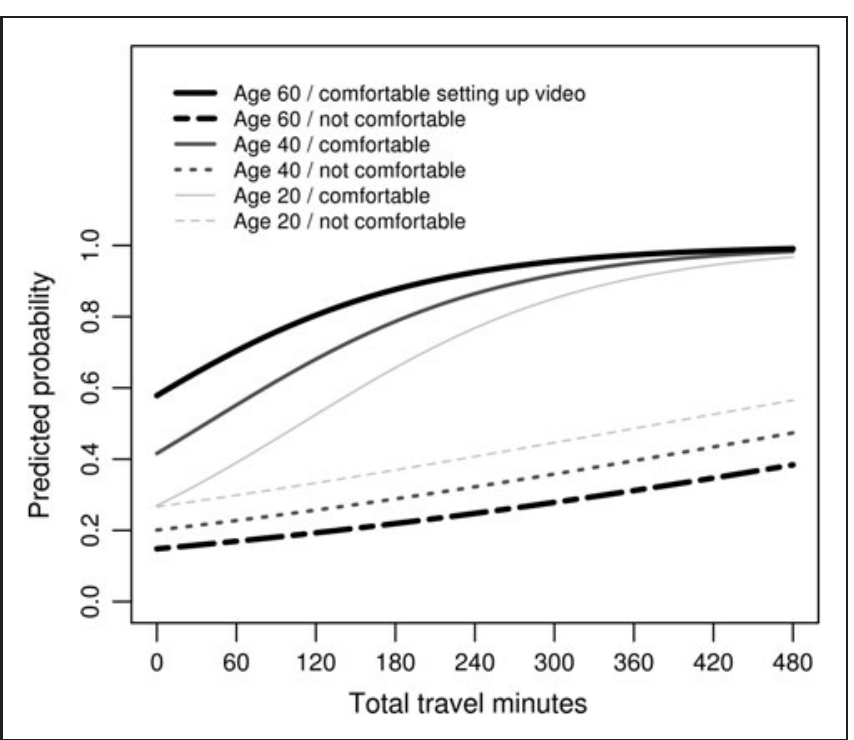

Fig. 2. Predicted probability to accept video invite based on three primary factors: patient age, comfort in setting up a video call, and travel distance (time).

of this, technology staff may be needed to assist with bringing on inexperienced patients who are willing to utilize in-home telehealth care.

The gap between high willingness to engage with a provider over video and the relatively little experience with the medium suggests that patients see video appointments as a feasible and desirable way to interact with their providers. They may be dependent, however, upon their provider to offer the service; they may not voluntarily ask for it because of a lack in fluency with related use cases. The survey focused on participant response to an invitation assuming an organization-driven offering and did not frame the questions as a patient-requested offering. For a complementary perspective, future research may wish to query demand by framing questions from a patient request perspective.

Although video appointments are a desirable concept for many in our sample, increasing desirability of the concept may prove difficult. Of the four primary concerns to address, prioritized by the correlation with average "disagreement" [see Fig. 1, questions (f), (i), and (b)], three of the four questions [(f), (i), and (h)] express concern not about logistics of connection, privacy, or security of the medium-all readily actionable items to address on the service provider's end-but about the general preferences of face-to-face over video communication. To shift perceptions about the medium may require deploying a focused communication strategy that addresses these perceptions, encouraging the use of the system on a trial basis, or simply allowing technology use behavior to catch up with what technology enables.

The primary factor for whether someone will accept an invitation is his or her comfort in setting up a call. This is not extremely surprising. If the technology is not understood or if someone is not technologically facile, the thought of having to set up a call could cause some anxiety. Most participants still prefer to speak with their provider in a traditional in-person appointment, even if a video call now makes some of the costs associated with travel avoidable.

This study did not gather socioeconomic data. The burden of the economic costs to travel for an appointment is relative to income, and because a video visit is a relatively low-cost alternative to travel, there is likely a financial trade-off decision being made. Further research on patient willingness may wish to gather socioeconomic data and conduct elasticity analysis on the relationship of income with the willingness to engage in a video appointment.

Participants indicated a fairly low likelihood that they would consider paying for a video appointment out of pocket, particularly those who live closer to the institution. Those who indicate likelihood are likely to live greater than $3 \mathrm{~h}$ away, which in many geographical locations would present out-of-state licensure barriers. In the current reimbursement environment, which is driven largely by government payer policies, there are few scenarios where a payer will reimburse providers for a video call between a provider and patients in their homes-most scenarios require the patient to be located in an accredited medical facility for a video appointment with providers.

However, as we look forward to a shift from fee-for-service to payfor-performance, some of the factors in the viability equation may change, but policy makers and payers should make sure to allow for "patient-centered" communication modalities. In the best of scenarios, the provider will be able to choose whatever method of communication best addresses the patient's needs, provides quality and value, and will be the most convenient to the patient.

This was not an elasticity study, and participants were not given a specific price point about which to decide whether a video appointment would be of enough value to them to pay for themselves. Further work needs to be done to understand patient tolerances for (self) payment in the current fee-for-service environment.

\section{LIMITATIONS}

Because the study did not target patients with any one condition or seen by any one specialty, we believe that it represents broad patient interests. These results are most representative of a population that is balanced with respect to distance to our facility, but we did not attempt to balance participants for age, race/ethnicity, or socioeconomics, and we recognize that the patient population from the single institution may not reflect the national population makeup. This potentiality has implications on prevalence of endpoint devices and broadband penetration and limits generalizations that can be made. This study did not seek to capture patient-specific economic data, which may have added additional insight into why participants indicated an interest to pay out of pocket for the services or to travel for care. This study did not seek access to patient health data, which may also have helped to add insight as to how they indicated interest. Further research is needed to assess if patient objectives (e.g., seeking a diagnosis, gaining clarification on test results, assessing a provider), particular conditions, or other measures of health might drive patient interests more than others. 


\section{PATIENT PERCEPTIONS OF IN-HOME VIDEO APPOINTMENTS}

\section{Conclusions}

It is evident that patient demand for video appointments from their homes is nascent, but that there is, nevertheless, a core of patients whose interest could be leveraged to help nurture mainstream usage. Interest in the service, once offered, is highly dependent on the patient's willingness and confidence to co-create the experience obtaining and setting up the components required for a video appointment on his or her end. Distance from the clinic is a definite motivator, but one that needs to be balanced with other economic costs to the patient. If the obstacles to creating and offering a reliable video appointment service can be overcome, for patients who have the interest, aptitude, and confidence, there exists an opportunity to co-create the broader experience and availability of video appointments.

\section{Acknowledgments}

Thanks to the Mayo Clinic Center for Innovation for supporting this study. We also want to thank Ms. Gladdie Hebl from Research and Academic Support Services at Mayo Clinic for her editorial assistance.

\section{Disclosure Statement}

No competing financial interest exists.

\section{REFERENCES}

1. Grigsby B, Brega AG, Bennett RE, Devore PA, Paulich MJ, Talkington SG, Floersch NR, Barton PL, Neal S, Araya TM, Loker JL, Krohn N, Grigsby J. The slow pace of interactive video telemedicine adoption: The perspective of telemedicine program administrators on physician participation. Telemed J E Health 2007;13:645-656.

2. Dixon $R F$, Stahl JE. A randomized trial of virtual visits in a general medicine practice. J Telemed Telecare 2009;15:115-117.
3. McDonald E, Lamb A, Grillo B, Lucas L, Miesfeldt S. Acceptability of telemedicine and other cancer genetic counseling models of service delivery in geographically remote settings. J Genet Couns 2013;23:221-228.

4. Agrell $H$, Dahlberg $S$, Jerant AF. Patients' perceptions regarding home telecare. Telemed J E Health 2000;6:409-415.

5. Levy S, Bradley DA, Morison MJ, Swanston MT, Harvey S. Future patient care: Tele-empowerment. J Telemed Telecare 2002;8(Suppl 2):52-54.

6. Cimperman M, Brencic MM, Trkman P, de Leonni Stanonik M. Older adults' perceptions of home telehealth services. Telemed J E Health 2013;19:786-790.

7. Whitten $P$, Love B. Patient and provider satisfaction with the use of telemedicine: Overview and rationale for cautious enthusiasm. J Postgrad Med 2005;51:294-300

8. Goodenough B, Cohn RJ. Parent attitudes to audio/visual telecommunications in childhood cancer: An Australian study. Telemed J E Health 2004;10(Suppl 2):S-15-S-25.

9. R Development Core Team. R: A language and environment for statistical computing. Available at www.R-project.org/ (last accessed July 5, 2013).

10. WWAMI (Washington W, Alaska, Montana, and Idaho) Rural Health Research Center. Rural-urban commuting area codes (RUCAs). Available at http:// depts.washington.edu/uwruca/ (last accessed June 5, 2013).

Address correspondence to:

Matthew R. Gardner, MBA, MDes

Center for Innovation Mayo Clinic

200 First Street SW Rochester, MN 55905

E-mail: gardner.matthew@mayo.edu

Received: February 17, 2014 Revised: July 14, 2014

Accepted: July 16, 2014 